\title{
A NOVEL AND SIMPLE SYNTHESIS OF SOME NEW AND KNOWN DIBENZO PHENAZINE AND QUINOXALINE DERIVATIVES USING LEAD DICHLORIDE
}

\author{
BAHADOR KARAMI*a,b AND SAEED KHODABAKHSHI \\ ${ }^{a}$ Department of Chemistry, Yasouj University, Yasouj, Zip Code: 75918-74831 P.O.Box 353, Iran \\ ${ }^{b}$ Department of Chemistry, Gachsaran Branch, Islamic Azad University, Gachsaran, Iran \\ 'Young Researchers Club, Gachsaran Branch, Islamic Azad University, Gachsaran, Iran
}

(Received: July 8, 2011 - Accepted: December 4, 2012)

\begin{abstract}
A simple method for the synthesis of phenazine and quinoxaline derivatives have been developed via a reaction of $o$-phenylenediamines and 1,2-dicarbonyl compounds or aryl glyoxals in the presence of lead dichloride in ethanol at room temperature. This method has many appealing attributes such as excellent yields, short reaction times, and simple work-up procedure.
\end{abstract}

Keywords: Phenazine, Quinoxaline, Lead dichloride, Catalyst.

\section{INTRODUCTION}

Phenazines and quinoxalines are important class of nitrogen heterocyclic compounds which they have significance both in chemistry and biology. ${ }^{1,2}$ Many phenazine compounds are found in nature and they are produced by bacteria such as Pseudomonas spp., Streptomyces spp., and Pantoea agglomerans. These phenazine natural products have been implicated in the virulence and competitive fitness of producing organisms. ${ }^{3,4}$ Quinoxaline and phenazine derivatives constitute the basis of many insecticides, antitumors, fungicides, herbicides, and receptor antagonists. ${ }^{5-9}$ Besides this, they are used in dyes ${ }^{10}$ building blocks for the synthesis of organic semiconductors ${ }^{11}$ chemically controllable switches ${ }^{12}$ cavitands ${ }^{13}$ DNA cleaving agents, ${ }^{14}$ dehydroannulenes, ${ }^{15}$ electrical-photochemical materials, ${ }^{16-18}$ and inhibitor for the corrosion of mild steel. ${ }^{19}$

A number of synthetic strategies have been developed for the preparation of substituted quinoxalines. ${ }^{20}$ Some of the existing methods suffer from disadvantages such as unsatisfactory product yields, harsh conditions, long reactions time, and critical product isolation procedures.

\section{RESULTS AND DISCUSSION}

In continuation of our studies on synthesis of organic compounds, ${ }^{21,22}$ we have now found that the lead dichloride $\left(\mathrm{PbCl}_{2}\right)$ can be used as an efficient and inexpensive catalyst for the condensation of 1,2-dicarbonyl compounds 1-3 or aryl glyoxals 4-8 with $o$-phenylenediamines 10 at room temperature to afford novel and known phenazines and quinoxalines 11-18 in high yields (Scheme $1)$.

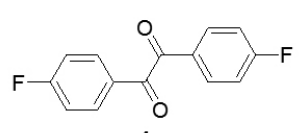

1<smiles>O=C(c1ccc2ccccc2c1)C(O)O</smiles>

4<smiles>COc1cccc(C(=O)C(O)O)c1</smiles>

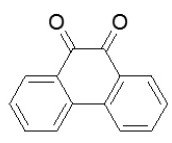

2

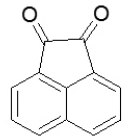

3<smiles>O=C(c1cccc([N+](=O)[O-])c1)C(O)O</smiles>

6<smiles>O=C(c1ccccc1)C(O)O</smiles>

7

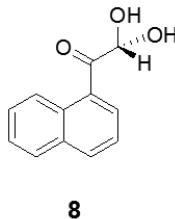

Figure 1: 1,2-dicarbonyl compounds.

For establishing the simple and suitable conditions to prepare of quinoxaline and phenazine derivatives using $\mathrm{PbCl}_{2}$ as Lewis acid catalyst, the treatment of 4,4'-difluoro-benzil 1 with $o$-phenylene diamine 10a was chosen as a model reaction (Table 2, Product 11a). Also, The Table 1 shows the obtained results for the several solvents which were tested in this experiment. It was observed that the condensation reaction can be efficiently carried out in ethanol as by adding $20 \mathrm{~mol} \%$ of the catalyst in a short time span of 25 minutes. The use of excess amounts of the catalyst does not show marked influence on product yield. Probably, the reason for this observation is the coordination of excessive catalyst to the diamine.

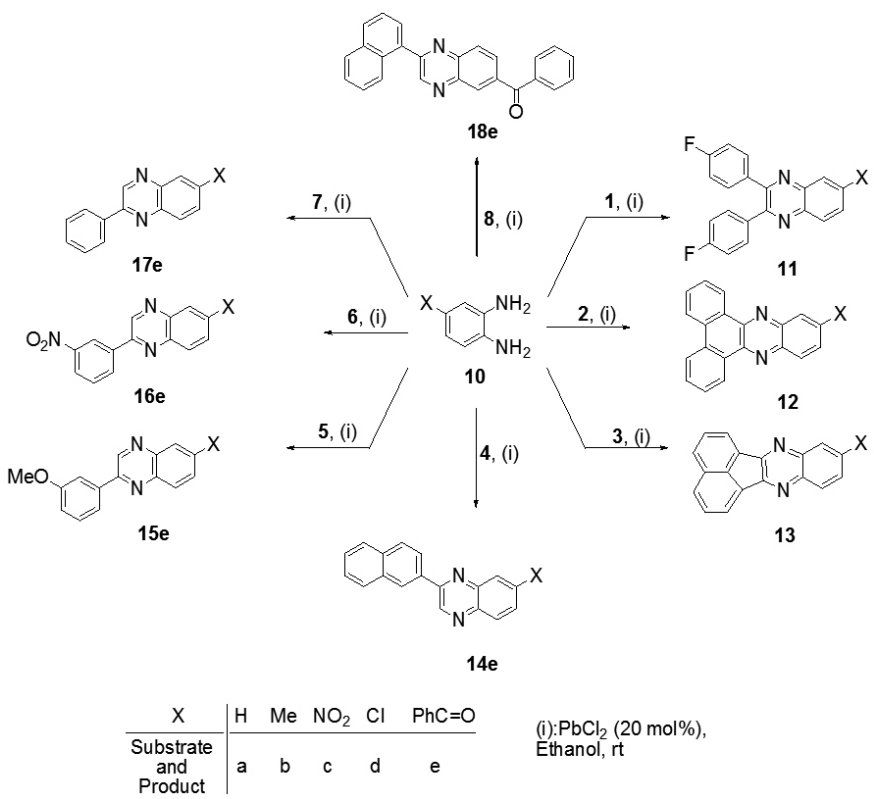

Scheme 1: The synthesis of quinoxaline and phenazine derivatives by the use of $\mathrm{PbCl}_{2}$.

Table 1: Solvent test for the synthesis of 11a using $\mathrm{PbCl}_{2}(20 \mathrm{~mol} \%)$ at room temperature.

\begin{tabular}{|c|c|c|c|}
\hline Entry & Solvent & Yield (\%) $^{\mathrm{a}}$ & Time (min) \\
\hline 1 & EtOH & 90 & 25 \\
\hline 2 & $\mathrm{MeOH}$ & 85 & 30 \\
\hline 3 & $\mathrm{H}_{2} \mathrm{O}$ & 35 & 300 \\
\hline 4 & $\mathrm{CH}_{2} \mathrm{Cl}_{2}$ & 60 & 180 \\
\hline 5 & $\mathrm{CH}_{3} \mathrm{Cl}$ & 65 & 180 \\
\hline
\end{tabular}

${ }^{a}$ Refers to isolated yields.

In order to prove the general applicability of this method, after optimizing the reaction conditions, we have treated different 1,2-dicarbonyl compounds or aryl glyoxals with $o$-phenylenediamines at room temperature in ethanol. The results are summarized in Table 2. 
Although good to excellent yields of the products have been obtained at room temperature, the reaction rate may be altered by varying the substituents on $o$-phenylenediamines $\mathbf{1 0}$ and also choosing the different 1,2-dicarbonyl compounds 1-3 or aryl glyoxals 4-8. For example, as can be seen in Table 2, electron-withdrawing group such as nitro on the diamine ring causes the reaction to proceed slowly (product $\mathbf{1 2 c}$ and $\mathbf{1 3 c}$ ).

Table 2: Synthesis of phenazine and quinoxaline derivatives using $\mathrm{PbCl}_{2}$ (20 $\mathrm{mol} \%)$ at room temperature.

\begin{tabular}{|c|c|c|c|}
\hline Product $^{\mathrm{a}}$ & Time (min) & Yield $^{\mathrm{b}}(\%)$ & M.p. ${ }^{\circ} \mathrm{C}$ \\
\hline 11a & 25 & 90 & $134-136^{23 a-c}$ \\
\hline 11b & 35 & 92 & $163-165^{23 a-c, 24 a}$ \\
\hline $11 \mathrm{c}$ & 50 & 90 & $176-177^{23 \mathrm{~b}, 24 \mathrm{a}-\mathrm{c}}$ \\
\hline $12 \mathrm{a}$ & 10 & 95 & $224-226^{25 a-d}$ \\
\hline $12 b$ & 10 & 95 & $217-219^{25 a-b}$ \\
\hline $12 \mathrm{c}$ & 320 & 86 & $260-261$ \\
\hline 12d & 25 & 90 & $246-247$ \\
\hline $12 \mathrm{e}$ & 15 & 97 & $245-247^{26}$ \\
\hline $13 \mathbf{a}$ & 20 & 93 & $237-239^{27 a-c}$ \\
\hline 13b & 20 & 95 & $230-232^{28 a-b}$ \\
\hline $13 \mathrm{c}$ & 340 & 85 & $320-321^{25 d}$ \\
\hline $13 d$ & 30 & 90 & $227-228$ \\
\hline $13 e$ & 20 & 95 & $250-251^{27 \mathrm{a}-\mathrm{c}}$ \\
\hline $14 \mathrm{e}$ & 25 & 92 & $193-194^{c}$ \\
\hline $15 e$ & 20 & 95 & $129-130^{c}$ \\
\hline $16 \mathrm{e}$ & 15 & 88 & $204-205^{c}$ \\
\hline $17 \mathrm{e}$ & 20 & 95 & $144-145^{\mathrm{c}}$ \\
\hline $18 \mathrm{e}$ & 20 & 93 & $166-167^{c}$ \\
\hline
\end{tabular}

${ }^{a}$ Identified by comparison with authentic samples. ${ }^{\mathrm{b}}$ Refers to isolated yields. ${ }^{\mathrm{c}}$ Novel compounds.

As can be seen in Table 3, In order to show the crude effect of $\mathrm{PbCl}_{2}$ as a heterogeneous and efficient catalyst, some representative reactions were also performed in the absence of $\mathrm{PbCl}_{2}$. Generally, based on the obtained results, it can be concluded that in the absence of the catalyst, the reaction proceeds slowly.

Table 3: Synthesis of some products in the absence of catalyst.

\begin{tabular}{|c|c|c|c|}
\hline Entry & Product & Yield (\%) & Time (min) \\
\hline 1 & 11a & 45 & 480 \\
\hline 2 & 13c & 50 & 720 \\
\hline 3 & 15e & 55 & 360 \\
\hline 4 & $\mathbf{1 7 e}$ & 40 & 480 \\
\hline
\end{tabular}

${ }^{\text {a }}$ Refers to isolated yields.

It is interesting to note that in this condensation reaction, 9,10-phenanthrene quinone $\mathbf{1 2}$ and acenaphtho quinone $\mathbf{1 3}$ are more active than the 4,4'-difluorobenzil 1. As can be seen in Scheme 2, as explanation of these rate differences, we believe that the steric and electronic effects can be key factors. In fact, benzil $\mathbf{1}$ adopts an S-trans (anti) conformer in usual form. Thus, as will be mentioned later (in suggested mechanism), for cyclocondensation of benzil 1 with o-phenylenediamines 10, benzil 1 should be rotated about C-C single bond to form the S-cis conformer as a favored transition state (Scheme 2, TS 1). Therefore, this lower activity observation of $\mathbf{1}$ than the other ones can be rationalized through the need to more activation energy of 1 .

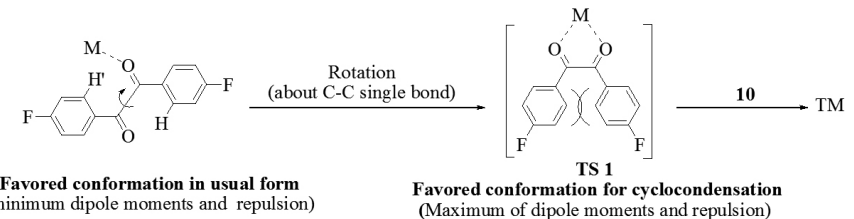

Scheme 2: Rotation about C-C single bond of benzil 1.
In the cases 9,10-phenanthrene quinone 12 and acenaphtho quinone $\mathbf{1 3}$, because of their rigid conformation (S-cis), the rotation about $\mathrm{C}$-C single bond is not required. In fact, both $\mathbf{2}$ and $\mathbf{3}$ have favored conformation for condensing with $o$-phenylenediamines $\mathbf{1 0}$ (Scheme 3, TS 2).

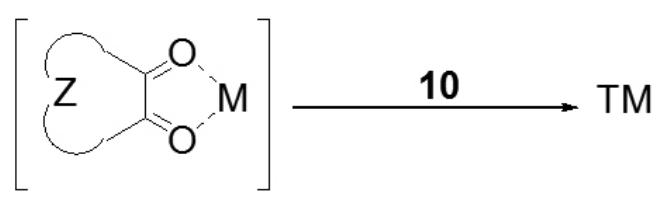

\section{TS 2. Rigid and favored conformation for usual form and Nu attack}

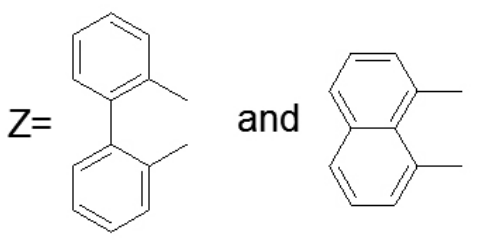

Scheme 3: Favored transition state for 12 and $\mathbf{1 3 .}$

Although the generally mechanistic details of this reaction are not yet fully understood, a feasible pathway, as indicated in Scheme 4, might involves the chelation of the carbonyl oxygen of dicarbonyls by lead in the first step. The importance of this chelation is the activation of carbonyl for amine attack. Also, the lead as transition metal Lewis acid plays a complex role in promoting the dehydration steps. The irreversibility of the reaction implies that reaction product is thermodynamically stable. However, the driving force for all of these reactions is cycloaromatization.

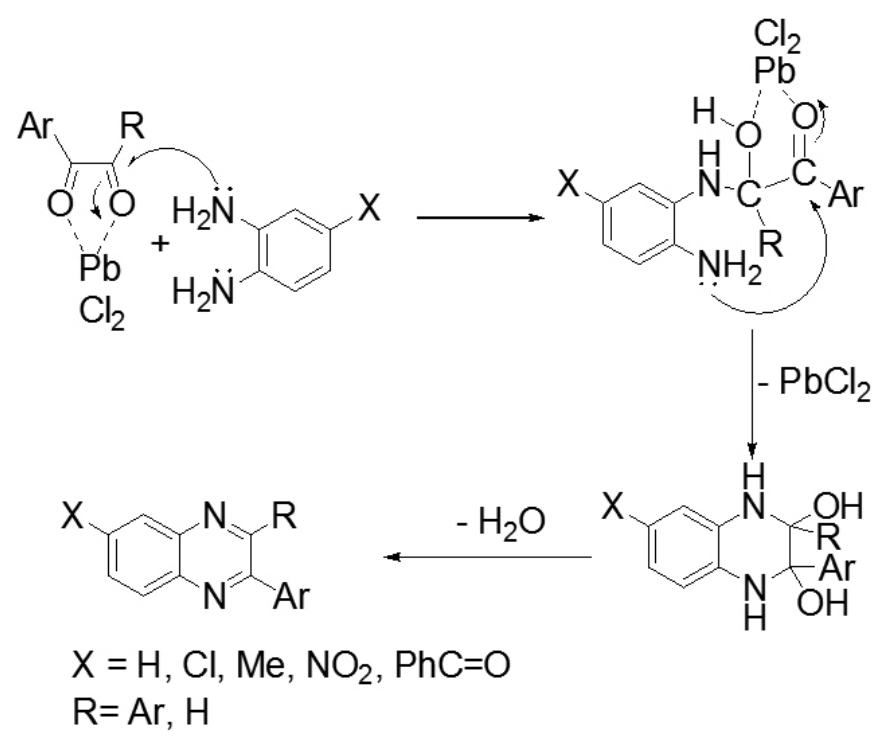

Scheme 4: Suggested mechanism for the synthesis of phenazines and quinoxalines using $\mathrm{PbCl}_{2}$.

\section{EXPERIMENTAL}

\subsection{General}

The commercial starting materials were purchased from Merck, Fluka and Aldrich. The reactions were monitored by TLC (silica gel $60 \mathrm{~F}_{254}$, hexane/ AcOEt). IR spectra were recorded on a FT-IR Shimadzu- 470 spectrometer and the ${ }^{1} \mathrm{H}$ NMR spectra were obtained on a Bruker-Instrument DPX-400 MHz Avance 2 model. The varioEl CHN Isfahan Industrial University was used for elemental analysis. All of the products (except novel compounds 14e-18e) were characterized by comparison of their spectra and physical data, with those reported in the literature..$^{23-28}$ 


\subsection{General procedure}

A mixture of 1,2- dicarbonyl compound or aryl glyoxal $(1 \mathrm{mmol})$, $o$-phenylenediamine $(1.1 \mathrm{mmol})$ and lead dichloride $(20 \mathrm{~mol} \%$ ) in ethanol $(5 \mathrm{~mL})$ was stirred at room temperature. The progress of the reaction was monitored by TLC (hexane/AcOEt, 3:7). After the completion of the reaction, the solid which separated was filtered and then recrystallized from ethanol to afford pure product.

3.3. Spectral data of some compounds

2,3-Bis(4-flouro-phenyl)quinoxaline (11a): ${ }^{1} \mathrm{H} \mathrm{NMR}\left(400 \mathrm{MHz}, \mathrm{CDC}_{3}\right)$ $\delta 7.97$ (dd, 2H, J 6.4, 3.6 Hz), 7.60 (dd, 2H, J 6.4, 3.2 Hz), 7.33-7.30 (m, 4H), $6.86(\mathrm{t}, 4 \mathrm{H}, J 8.8 \mathrm{~Hz}){ }^{13} \mathrm{C}$ NMR $\left(100 \mathrm{MHz}, \mathrm{CDC}_{1}\right) \delta 161.99,152.20,141.23$, 135.02, 131.82, 131.74, 130.23, 129.16, 115.65, 115.43; IR (KBr): v 3061, $1599,1555,1511,1344,1225,839,786 \mathrm{~cm}^{-1}$

2,3-Bis(4-flouro-phenyl)-6-methylquinoxaline (11b): ${ }^{1} \mathrm{H}$ NMR $(400$ $\left.\mathrm{MHz}, \mathrm{CDC1}_{3}\right) \delta 6.58(\mathrm{t}, 4 \mathrm{H}, J 8.8 \mathrm{~Hz}), 2.43(\mathrm{~s}, 3 \mathrm{H}), 7.85(\mathrm{~d}, 1 \mathrm{H}, J 8.8 \mathrm{~Hz})$, $7.73(\mathrm{~s}, 1 \mathrm{H}), 7.42(\mathrm{~d}, 1 \mathrm{H}, J 8.8 \mathrm{~Hz}), 7.30(\mathrm{dd}, 4 \mathrm{H}, J 8.00,5.2 \mathrm{~Hz}) ;{ }^{13} \mathrm{C} \mathrm{NMR}$ $\left(100 \mathrm{MHz}, \mathrm{CDC} 1_{3}\right): \delta 161.89,152.05,151.29,141.28,140.84,139.69,135.16$, 135.13, 132.59, 131.77, 131.72, 131.69, 128.65, 127.96, 115.59, 115.37, 21.94; IR (KBr): $v 2925,2580,1657,1597,1264,1159,833,696 \mathrm{~cm}^{-1}$.

Dibenzola,clphenazine (12a): ${ }^{1} \mathrm{H}$ NMR $\left(400 \mathrm{MHz}, \mathrm{CDC}_{3}\right): \delta 9.18(\mathrm{~d}$, $2 \mathrm{H}, J 7.6 \mathrm{~Hz}), 8.34(\mathrm{~d}, 2 \mathrm{H}, J 8 \mathrm{~Hz}), 8.12(\mathrm{dd}, 2 \mathrm{H}, J 6.4,3.6 \mathrm{~Hz}), 7.66-7.51(\mathrm{~m}$, $6 \mathrm{H}) ;{ }^{13} \mathrm{C}$ NMR (100 MHz, $\left.\mathrm{CDC1}_{3}\right): \delta 143.54,143.28,133.15,131.42,130.88$, 130.57, 129.04, 127.38, 124.03; IR (KBr): v 3055, 1600, 1490, 1350, 760,720 $\mathrm{cm}^{-1}$.

11-Methyl-dibenzo[a,clphenazine (12b): ${ }^{1} \mathrm{H}$ NMR (400 $\left.\mathrm{MHz}, \mathrm{CDC}_{3}\right)$ : $\delta 9.14(2 \mathrm{H}, \mathrm{dd}, J 6.00,1.6 \mathrm{~Hz}), 8.32(\mathrm{~d}, 2 \mathrm{H}, J 8 \mathrm{~Hz}), 7.97(\mathrm{~d}, 1 \mathrm{H}, J 8.4 \mathrm{~Hz})$, $7.58(\mathrm{~s}, 1 \mathrm{H}), 7.53-7.52(\mathrm{~m}, 5 \mathrm{H}), 2.54(\mathrm{~s}, 3 \mathrm{H}) ;{ }^{13} \mathrm{C} \mathrm{NMR}\left(100 \mathrm{MHz}, \mathrm{CDC1}_{3}\right)$ : $\delta 143.29,143.27,142.72,141.81,141.41,133.45,133.06,132.87,131.49$, $131.45,131.20,131.07,130.01,129.10,128.92,127.29,127.15,123.95,23.20$; IR (KBr): $v$ 3055, 2910,1620, 1500, 1350, 760, $720 \mathrm{~cm}^{-1}$.

11-Benzoyl-dibenzo/a,c/phenazine (12e): ${ }^{1} \mathrm{H} \mathrm{NMR}\left(\mathrm{CDCl}_{3}, 400 \mathrm{MHz}\right): \mathrm{d}$ $9.43(\mathrm{dd}, 1 \mathrm{H}, J 8 \mathrm{~Hz}, 1.2 \mathrm{~Hz}), 9.35$ (dd, $1 \mathrm{H}, J 8 \mathrm{~Hz}, 1.2 \mathrm{~Hz}), 8.70$ (d, 1H, $J 1.6$ $\mathrm{Hz}), 8.58(\mathrm{~d}, 2 \mathrm{H}, J 8 \mathrm{~Hz}), 8.44(\mathrm{~d}, 1 \mathrm{H}, J 8.8 \mathrm{~Hz}), 8.55(\mathrm{dd}, 1 \mathrm{H}, J 8.8 \mathrm{~Hz}, 2 \mathrm{~Hz})$, 7.99-7.97 (m, 2H), 7.87-7.68 (m, 5H), 7.60 (t, $2 \mathrm{H}, J 8 \mathrm{~Hz}) ;{ }^{13} \mathrm{C} \mathrm{NMR}\left(\mathrm{CDCl}_{3}\right.$, 100MHz): d 196.07, 184.81, 153.70, 143.74, 143.45, 141.05, 137.92, 137.38, $132.95,132.84,132.48,132.18,130.99,130.75,130.23,129.94,129.40$, 128.58, 128.12, 126.69, 126.36, 123.01; IR (KBr): v 3050, 1650, 1600, 1445, $1320 \mathrm{~cm}^{-1}$.; Anal. Calcd. for $\mathrm{C}_{27} \mathrm{H}_{16} \mathrm{~N}_{2} \mathrm{O}$ : C, 84.36; H, 4.20; N, 7.29. Found: $\mathrm{C}$, 84.48, H, 4.183, N, 7.375.

Acenaphtho[1,2-b] quinoxaline (13a): ${ }^{1} \mathrm{H}$ NMR $\left(400 \mathrm{MHz}, \mathrm{CDC}_{3}\right): \delta$ 8.21 (d, 2H, J 6.8 Hz), 8.02 (dd, 2H, J 6.2, 3.2 Hz), 7.90 (d, 2H, J 8.4 Hz), 7.65 (t, $2 \mathrm{H}, J 7 \mathrm{~Hz}), 7.57(\mathrm{dd}, 2 \mathrm{H}, J 6.4,3.6 \mathrm{~Hz}) ;{ }^{13} \mathrm{C}$ NMR $\left(100 \mathrm{MHz}, \mathrm{CDC}_{3}\right)$ : $\delta 155.19,142.39,137.60,132.92,131.10,130.47,130.59,130.36,129.78$, 122.96; IR (KBr): v 3050, 1610, 1430, 1300, 830, $760 \mathrm{~cm}^{-1}$.

9-Methyl-acenaphtho[1,2-b]quinoxaline (13b): ${ }^{1} \mathrm{H}$ NMR (400 MHz, $\left.\mathrm{CDC1}_{3}\right): \delta 8.21(\mathrm{t}, 2 \mathrm{H}, J 6.4 \mathrm{~Hz}), 7.90(\mathrm{dd}, 3 \mathrm{H}, J 8.2 \mathrm{~Hz}, 3.2 \mathrm{~Hz}), 7.79(\mathrm{~s}, 1 \mathrm{H})$, $7.64(\mathrm{t}, 2 \mathrm{H}, J 7.4 \mathrm{~Hz}), 7.40(\mathrm{dd}, 1 \mathrm{H}, J 8.4,1.6 \mathrm{~Hz}), 2.43(\mathrm{~s}, 3 \mathrm{H}) ;{ }^{13} \mathrm{C} \mathrm{NMR}$ $\left(100 \mathrm{MHz}, \mathrm{CDC}_{1}\right): \delta 155.15,154.44,142.38,140.82,140.71,137.35,133.08$, 132.44, 131.06, 130.46, 130.31, 130.21, 129.89, 129.72, 122.83, 122.68, 22.94; IR (KBr): $v 3055,2910,1610,1415,1300,810,790 \mathrm{~cm}^{-1}$.

9-Benzoylacenaphtho[1,2-b]quinoxaline (13e): ${ }^{1} \mathrm{H}$ NMR $\left(\mathrm{CDCl}_{3}\right.$, $400 \mathrm{MHz}): \mathrm{d} 8.61(\mathrm{~d}, 1 \mathrm{H}, J 1.6 \mathrm{~Hz}), 8.50(\mathrm{~d}, 1 \mathrm{H}, J 6.8 \mathrm{~Hz}), 8.44(\mathrm{~d}, 1 \mathrm{H}, J 6.8$ $\mathrm{Hz}), 8.34(\mathrm{~d}, 1 \mathrm{H}, J 8.8 \mathrm{~Hz}), 8.28(\mathrm{dd}, 1 \mathrm{H}, J 8.6 \mathrm{~Hz}, 2 \mathrm{~Hz}), 8.18(\mathrm{dd}, 2 \mathrm{H}, J 8$ $\mathrm{Hz}, 6 \mathrm{~Hz}), 7.96-786(\mathrm{~m}, 4 \mathrm{H}), 7.67(\mathrm{t}, 1 \mathrm{H}, J 7.6 \mathrm{~Hz}), 7.57(\mathrm{t}, 2 \mathrm{H}, J 7.6 \mathrm{~Hz}) ; \mathrm{IR}$ (KBr): v 3038, 1646, 1595, 1437, $1300 \mathrm{~cm}^{-1}$.; Anal. Calcd for $\mathrm{C}_{25} \mathrm{H}_{14} \mathrm{~N}_{2} \mathrm{O}: \mathrm{C}$, 83.78; H, 3.94; N, 7.82. Found: C, 83.46, H, 3.745, N, 7.607.

3-(2-Naphthyl)-7-benzoyl-quinoxaline (14e): ${ }^{1} \mathrm{H}$ NMR $(400 \mathrm{MHz}$, $\left.\mathrm{CDCl}_{3}\right)$ : d $9.59(\mathrm{~s}, 1 \mathrm{H}), 8.74(\mathrm{~s}, 1 \mathrm{H}), 8.58(\mathrm{~s}, 1 \mathrm{H}), 8.43(\mathrm{dd}, 1 \mathrm{H}, J 8.6,1.6 \mathrm{~Hz})$, $8.32(\mathrm{~s}, 2 \mathrm{H}), 8.09-8.05(\mathrm{~m}, 2 \mathrm{H}), 7.95-7.93(\mathrm{~m}, 3 \mathrm{H}), 7.68(\mathrm{t}, 1 \mathrm{H}, J 7.6 \mathrm{~Hz}), 7.64-$ 7.55 (m, 4H); ${ }^{13} \mathrm{C}$ NMR $(100 \mathrm{MHz}, \mathrm{CDCl}): \mathrm{d} 195.68,153.21,145.21,144.58$, $144.28,140.63,137.89,137.18,134.44,133.56,133.37,132.95,132.68$, $132.39,130.34,130.18,130.09,129.25,129.05,128.57,127.89,127.70$, 126.89, 124.41; IR (KBr): v 3090, 1649, 1309; Anal. Calcd for $\mathrm{C}_{25} \mathrm{H}_{16} \mathrm{~N}_{2} \mathrm{O}: \mathrm{C}$, $83.31 \mathrm{~cm}^{-1} ; \mathrm{H}, 4.47 ; \mathrm{N}, 7.77$. Found: C, 83.45, H, 4.40, N, 7.81 .

3-(3-Methoxyphenyl)-7-benzoyl-quinoxaline (15e): ${ }^{1} \mathrm{H}$ NMR $(400 \mathrm{MHz}$, $\left.\mathrm{CDCl}_{3}\right)$ : d $9.42(\mathrm{~s}, 1 \mathrm{H}), 8.52(\mathrm{~s}, 1 \mathrm{H}), 8.292(\mathrm{~s}, 2 \mathrm{H}), 7.93(\mathrm{~d}, 2 \mathrm{H}, J 7.2 \mathrm{~Hz}), 7.83$ $(\mathrm{t}, 2 \mathrm{H}, J 7.6 \mathrm{~Hz}), 7.67(\mathrm{~d}, 1 \mathrm{H}, J 7.6 \mathrm{~Hz}), 7.58-7.51(\mathrm{~m}, 3 \mathrm{H}), 7.14(\mathrm{dd}, 1 \mathrm{H}, J 8$, $2 \mathrm{~Hz}), 3.98$ (s, 3H); ${ }^{13} \mathrm{C}$ NMR (CDCl3, $\left.100 \mathrm{MHz}\right): \mathrm{d} 195.67,160.44,153.13$, $144.51,144.13,140.72,137.94,137.65,137.15,132.90,132.33,130.32$, $130.27,130.17,130.11,128.56,120.13,116.88,112.86,55.54$; IR $(\mathrm{KBr}): v$ 3090, 2950, 1649, 1598, 1329, $1297 \mathrm{~cm}^{-1}$; Anal. Calcd for $\mathrm{C}_{22} \mathrm{H}_{16} \mathrm{~N}_{2} \mathrm{O}_{2}$ : C, 77.63 ; H, 4.74; N, 8.23. Found: C, 77.80; H, 4.61; N, 8.15.

3-(3-Nitrophenyl)-7-benzoyl-quinoxaline (16e): ${ }^{1} \mathrm{H}$ NMR $(400 \mathrm{MHz}$,
$\left.\mathrm{CDCl}_{3}\right): \mathrm{d} 9.51(\mathrm{~s}, 1 \mathrm{H}), 9.18(\mathrm{~s}, 1 \mathrm{H}), 8.64(\mathrm{~d}, 1 \mathrm{H}, J 7.6 \mathrm{~Hz}), 8.55(\mathrm{~s}, 1 \mathrm{H}), 8.43$ $(\mathrm{dd}, 1 \mathrm{H}, J 8,1.2 \mathrm{~Hz}), 8.34(\mathrm{~s}, 2 \mathrm{H}), 7.94(\mathrm{~d}, 2 \mathrm{H}, J 7.2 \mathrm{~Hz}), 7.83(\mathrm{t}, 1 \mathrm{H}, J 8$ $\mathrm{Hz}), 7.69(\mathrm{t}, 1 \mathrm{H}, 7.2 \mathrm{~Hz}), 7.58(\mathrm{t}, 2 \mathrm{H}, 7.6 \mathrm{~Hz}) ;{ }^{13} \mathrm{C} \mathrm{NMR}\left(100 \mathrm{MHz}, \mathrm{CDCl}_{3}\right)$ : d $195.47,150.61,149.09,143.88,143.57,141.22,138.79,137.98,136.93$, $133.27,133.08,133.24,130.79,130.37,130.24,130.19,128.63,125.21$, 122.68; IR (KBr): $v$ 3090, 1649, 1533, 1348, $1297 \mathrm{~cm}^{-1}$; Anal. Calcd for $\mathrm{C}_{21} \mathrm{H}_{13} \mathrm{~N}_{3} \mathrm{O}_{3}: \mathrm{C}, 70.98 ; \mathrm{H}, 3.69 ; \mathrm{N}, 11.83$. Found: C, 71.16; H, 3.56; N, 11.90.

3-Phenyl-7-benzoyl-quinoxaline (17e): ' $\mathrm{H}$ NMR $\left(\mathrm{CDCl}_{3}, 400 \mathrm{MHz}\right)$ : d $9.43(\mathrm{~s}, 1 \mathrm{H}), 8.51(\mathrm{~s}, 1 \mathrm{H}), 8.28-8.25(\mathrm{~m}, 4 \mathrm{H}), 7.29(\mathrm{~s}, 1 \mathrm{H}), 7.91(\mathrm{~d}, 1 \mathrm{H}, J$ $1.2 \mathrm{~Hz}), 7.68-7.53(\mathrm{~m}, 6 \mathrm{H}) ;{ }^{13} \mathrm{C}$ NMR $\left(\mathrm{CDCl}_{3}, 100 \mathrm{MHz}\right)$ : d 195.63, 153.31, $144.41,144.16,140.63,137.88,137.12,136.24,132.89,132.33,130.82$ $130.26,130.16,130.09,129.30,128.54,127.77,127.60$; IR (KBr): v 3053, 1650, 1595, 1454, $1294 \mathrm{~cm}^{-1}$; Anal. Calcd for $\mathrm{C}_{21} \mathrm{H}_{14} \mathrm{~N}_{2} \mathrm{O}: \mathrm{C}, 81.27$; H, 4.55; N, 9.03. Found: C, 81.50, H, 4.516, N, 9.070.

3-Naphthyl-7-benzoyl-quinoxaline (18e): ${ }^{1} \mathrm{H}$ NMR $\left(\mathrm{CDCl}^{4} 400 \mathrm{MHz}\right): \mathrm{d}$ $9.31(\mathrm{~s}, 1 \mathrm{H}), 8.59(\mathrm{~s}, 1 \mathrm{H}), 8.35(\mathrm{~s}, 2 \mathrm{H}), 8.23(\mathrm{~d}, 1 \mathrm{H}, J 9.2 \mathrm{~Hz}), 8.06(\mathrm{~d}, 1 \mathrm{H}, J 8.4$ $\mathrm{Hz}), 8.01(\mathrm{~d}, 1 \mathrm{H}, J 6.4 \mathrm{~Hz}), 7.94(\mathrm{~d}, 2 \mathrm{H}, J 8 \mathrm{~Hz}), 7.48(\mathrm{~d}, 1 \mathrm{H}, J 7.2 \mathrm{~Hz}), 7.68$ (t, $2 \mathrm{H}, J 7.6 \mathrm{~Hz}), 7.60-7.53$ (m, 4H); ${ }^{13} \mathrm{C} \mathrm{NMR}\left(\mathrm{CDCl}_{3} 100 \mathrm{MHz}\right): \mathrm{d} 195.65$, 155.97, 147.77, 144, 140.41, 138.31, 137.08, 134.61, 134.07, 133.01, 132.38, $131.01,130.67,130.40,130.23,130.14,128.90,128.79,128.60,127.46$, $126.89,126.54,125.46,124.89$; IR (KBr): v 3050, 1697, 1651, 1446,1289 cm '.; Anal. Calcd for $\mathrm{C}_{25} \mathrm{H}_{16} \mathrm{~N}_{2} \mathrm{O}$ : C, 83.31; H, 4.47; N, 7.77. Found: C, 82.63, H, $4.523, \mathrm{~N}, 6.903$

\section{CONCLUSION}

In summary, we have presented a new application of lead dichloride $\left(\mathrm{PbCl}_{2}\right)$ as an effective and heterogeneous catalyst for the synthesis of many quinoxalines and phenazines based on the condensation of 1,2-dicarbonyl compounds with $o$-phenylenediamines under mild reaction conditions. Availability and stability of the catalyst, simple work-up procedure and the high yields, short reaction times, and mild reaction conditions make this method a valid contribution to the existing methodologies.

\section{ACKNOWLEDGEMENTS}

The authors gratefully acknowledge partial support of this work by the Yasouj University Iran.

\section{REFERENCES}

1. (a) W. Zhu, M. Dai, Y. Xu, X. Qian, Bioorg. Med. Chem. 16, 3255, (2008). (b) M. M. F. Ismail, Y. A. Ammar, M. K. H. Ibrahim, S. A. Elzahaby, S. S. Mahmoud, Arzeimittel Forschung/ Drug Res. 55, 738, (2006).

2. (a) X. Hui, J. Desrivot, C. Bories, P. M. Loiseau, X. Franck, R. Hocquemiller, B. Figadere, Bioorg. Med. Chem. Lett. 16, 815, (2006). (b) S. A. Kotharkar, D. B. Shinde, J. Iran. Chem. Soc. 3, 267, (2006).

3. P. Roy, B. K. Ghorai, Beilstein J. Org. Chem. 6, 1, (2010).

4. (a) J. M. Turner, A. J. Messenger, Adv. Microb. Physiol. 27, 211, (1986). (b) M. McDonald, D. V. Mavrodi, J. Am. Chem. Soc. 38, 9459, (2001),

5. H. Budzikiewicz, FEMS Microbiol. Lett. 104, 209, (1993).

6. N. Sato, vol. 6, in: A. R. Katritzky, C. W. Rees, E. F. V. Scriven (Eds.), Comprehensive Heterocyclic Chemistry II, vol. 6, Pergamon, Oxford, 1996, p. 233.

7. A. Gazit, H. App, G. McMahon, J. Chen, A. Levitzki, F. D. Bohmer, J. Med. Chem. 39, 2170, (1996).

8. (a) D. Bandyopadhyay, S. Mukherjee, R. R. Rodriguez, B. K. Banik, Molecules 15, 4207, (2010). (b) G. Sakata, K. Makino, Y. Kurasawa, Heterocycles 27, 2481, (1998).

9. (a) L. E. Seitz, W. J. Suling, R. C. J. Reynolds, J. Med. Chem. 45 , 5604, (2002). (b) A. C. Ahoya, B. Daouda, R. Bouhfid, A. Hancali, M. Bousminna, A. Zerzouf, R. El Aouad, E. M, Essassi, Arkivoc (ii), 217, (2011).

10. A. Katoh, T. Yoshida, J. Ohkanda, Heterocycles 52, 911, (2000).

11. S. Dailey, W. J. Feast, R. J. Peace, I. C. Sage, S. Till, E. L. Wood, J. Mater. Chem. 11, 2238 (2001)

12. M. J. Crossley, L. A. Johnston, Chem. Commun. 1122, (2002).

13. J. L. Sessler, H. Maeda, T. Mizuno, V. M. Lynch, H. Furuta, J. Am. Chem. Soc. $124,13474,(2002)$

14. T. Yamaguchi, S. Matsumoto, K. Watanabe, Tetrahedron Lett. 39, 8311, (1998).

15. O. Sascha, F. Rudiger, Synlett. 1509, (2004).

16. T. Yamamoto, B. L. Lee, H. Kokubo, H. Kishida, K. Hirota, T. 
Wakabayashi, H. Okamoto, Macromol. Rapid. Commun. 24, 440, (2003). 17. I. Nurulla, I. Yamaguchi, T. Yamamoto, Polym. Bull. 44, 231, (2000).

18. T. Yamamoto, B. L. Lee, H. Kokubo, H. Kishida, K. Hirota, T. Wakabayashi, H. Okamoto, Macromol. Rapid. Commun. 24, 440, (2003).

19. I. B. Obot, N. O. Obi-Egbedi, Mater. Chem. Phys. 122, 325, (2010).

20. (a) B. J. Reddy, M.C. S. Reddy, J. Chil. Chem. Soc. 55, 483, (2010). (b) V. Jeena, R. S. Robinson, Beilstein J. Org. Chem. 5, 1, (2009). (c) D. J. Brown, Quinoxalines: Supplement II, in: E.C. Taylor, P. Wipf (Eds.), The Chemistry of Heterocyclic Compounds, John Wiley\&Sons, New Jersey, 2004.

21. B. Karami, S. Khodabakhshi, K. Eskandari, Tetrahedron Lett. 53, 1445, (2012). (b) B. Karami, Z. Haghighijou, M. Farahi, S. Khodabakhshi, Phosphorus Sulfur Silicon Relat. Elem. 187, 754, (2012). (c) B. Karami, V. Ghashghaee, S. Khodabakhshi, Catal. Commun. 20, 71, (2012).

22. (a) B. Karami, S. Khodabakhshi, N. Safikhani, A. Arami, Bull. Korean Chem. Soc. 33, 123, (2012). (b) B. Karami, S. J. Hoseini, K. Eskandari, A. Ghasemi, H. Nasrabadi, Catal. Sci. Technol. 2, 331, (2012). (c) B. Karami, M. Farahi, S. Khodabakhshi, Helv. Chim. Acta 95, 455, (2012).

23. (a) M. M. Heravi, M. H. Tehrani, K. Bakhtiari, N. M. Javadi, H. A. Oskooie, Arkivoc (xvi), 16, (2006). (b) M. M. Heravi, K. Bakhtiari, F. F. Bamoharram, M. H. Tehrani, Monatsh. Fur. Chim. 138, 465, (2007). (c) C. Ochoa, J. Rodriguez, J. Heterocycl. Chem. 34, 1053, (1997).
24. (a) B. B. F. Mirjalili, A. Bamoniri, A. Akbari, Chem. Heterocycl. Compd. 47, 487, (2011). (b) M. Hoddapour, Indian J. Chem. 3, 87, (2011). (c) M. M. Heravi, M. hosseini, H. A. Oskooie, B. Baghernejad, J. Korean Chem. Soc. 55, 235, (2011). (d) A. Zare, A. Hasaninejad, A. Parhami, A. R. Moosavi-Zare, F. Khedri, Z. Parsaee, M. Abdolalipoor-Saretoli, M. Khedri, M. Roshankar, H. Deisi, J. Serb. Chem. Soc. 75, 1315, (2010). (e) V. K. Akkilagunta, V. P. Reddy, R. R. Kakulapati, Synlett 17, 2571, (2010).

25. (a) K. Niknam, D. Saberi, M. Mohagheghnejad, Molecules 14, 1915, (2009). (b) L. Carlier, M.1 Baron, A. Chamayou, G. Couarraze, Tetrahedron Lett. 52, 4686, (2011). (c) G. Kaupp, M. R. Naimi-Jamal, Eur. J. Org. Chem, 8, 1368, (2002). (d) K. Niknam, M. A. Zolfigol, Z. Tavakoli, Z. Heydari, J. Chin. Chem. Soc. 55, 1373, (2008).

26. A. N. Dobrodei, A. V. Eltsov, Russ. J. Gen. Chem. 68, 620, (1998).

27. (a) H.-Y. Lu, S.-H. Yang, J. Deng, Z.-H. Zhang, Australian J. Chem. 63, 1290, (2010). (b) H. Alinezhad, M. Tajbakhsh, F. Salehian, P. Biparva, Bull. Korean Chem. Soc, 32, 3720, (2011). (c) J. T. Hou, Y. H. Liu, Z. H. Zhung, J. Heterocyclic Chem. 47, 703, (2010).

28. (a) A. Boido, L. Vazzana, F. Sparatore, Farmaco 49, 97, (2010). (b) B. Karami, S. Khodabakhshi, J. Serb. Chem. Soc. 76, 1191, (2011). 